\title{
The Nomadic People Optimizer applied to the economic dispatch problem with prohibited operating zones
}

\author{
Lucas Santiago Nepomuceno \\ Department of Energy \\ Federal University of Juiz de Fora \\ Juiz de Fora, Minas Gerais, Brazil \\ lucas.nepomuceno@engenharia.ufjf.br
}

\author{
Gabriel Schreider da Silva \\ Department of Energy \\ Federal University of Juiz de Fora \\ Juiz de Fora, Minas Gerais, Brazil \\ gabriel.schreider2016@engenharia.ufjf.br
}

\author{
Edimar Jose de Oliveira \\ Department of Energy \\ Federal University of Juiz de Fora \\ Juiz de Fora, Minas Gerais, Brazil \\ edimar.oliveira@ufjf.edu.br
}

\author{
Arthur Neves de Paula \\ Department of Energy \\ Federal University of Juiz de Fora \\ Juiz de Fora, Minas Gerais, Brazil \\ arthur.paula@engenharia.ufjf.br
}

\author{
Edmarcio Antonio Belati \\ CECS \\ Federal University of $A B C$ \\ Santo André, São Paulo, Brazil \\ edmarcio.belati@ufabc.edu.br
}

\begin{abstract}
This work proposes the application of the Nomadic People Optimizer (NPO) to solve the economic dispatch problem considering Prohibitive Operating Zones (POZ). The NPO is a swarm-based metaheuristic recently introduced in the literature and still under-explored. In addition, the POZ increase the difficulties to find the optimal solution of the economic dispatch problem. The performance of the proposed methodology is compared with others metaheuristics present in the literature. Also, a sensibility analysis was performed. The NPO performed better than Ant Colony Optimization (ACO) and Whale Optimization Algorithm (WOA) metaheuristics in solving the problem.
\end{abstract}

Keywords: Economic dispatch. Nomadic People Optimizer. Prohibited operating zones. Metaheuristics. Bio-inspired optimization.

\section{INTRODUCTION}

The fossil fuels, widely used in thermal power plants (TPP), have a high-cost value when compared to other primary sources used in other forms of electricity generation, such as solar and wind.An aggravating factor for this situation is the currently hydric crisis that Brazil is facing, in which the water reservoir of the hydraulic power plants - main source of electricity generation in the country - reached the lowest level in the last 91 years. In this scenario, the use of TPPs to generate electricity is intensified to keep the load supply of the National Interconnected System, which causes a significant increase of the generation cost of the system. The country broke the historical record of electricity generation through TPP, producing 18787.78 MW in average on May 31, 2021, a level never before reached [1]. Therefore, it is clear the importance of optimal dispatch of TPPs aiming to minimizing the generation cost.

The economic dispatch (ED) problem is characterized by the TPPs dispatch aiming to minimize the system's generation cost, i.e., to determine the active power outputs of TPPs so that the generation cost is as low as possible [2]. Therefore, the ED problem consists in an optimization problem where the objective function $(\mathrm{OF})$ seeks to minimize the generation cost. Also, there is some constrains that must be respected [3]. These constrains are related to the maximum and minimum generation limits, due to the operational limits of the generators, and the system load demand plus the losses [4].

In most cases involving the ED problem, an approximate quadratic function relates the generation costs to the active power dispatch, constituting the $\mathrm{OF}[5,6]$. However, this representation of the ED problem is not very realistic, as it disregards the practical constraints of the TPP generator machines. To achieve a more realistic representation of the ED problem, prohibitive operating zones (POZs) can be considered, which are related to auxiliary services of TPPs, among others, such as boilers and feed pumps, which define operating regions unstable that should be avoided [7]. With the inclusion of the POZs, more inequalities are added to the set of constraints of the ED problem and the cost curve of a TPP is now interpreted as a curve determined by these inequalities, being divided into several isolated sub-regions, which form multiple spaces of decision, this makes ED a nonconvex and discontinuous problem, causing a large increase in its complexity [7-9].

The metaheuristics are stochastic optimization methods capable of solving several problems in a generic way [10]. In the field of power systems, optimization via the metaheuristic technique is widely used in problems such as transmission and distribution expansion planning, economic dispatch, power flow optimization, load forecasting, among others [11]. The metaheuristics are generally simple and easy to implement, in addition to being flexible to modify their structure and param- 
eters [12]. Obtaining the optimal solution is not guaranteed when using a meta-heuristic to solve a problem. However, excellent quality solutions can be found at a relatively low computational cost $[12,13]$.

As already mentioned, the ED problem taking into account more realistic conditions, such as POZs, is a complex mathematical programming problem, due to its non-convexity and discontinuities. Therefore, to solve this type of problem, appropriate optimization techniques have been widely investigated in the specialized literature $[3,8,14,15]$.

In [3] the authors propose the use of the bio-inspired metaheuristic called Bat Algorithm (BA) to solve the problem of ED with insertion of discontinuities arising from the consideration of POZs.

In [8], the authors also applied the BA technique in the ED realistically modeled with the representation of practical constraints. However, in this work an improved version of this meta-heuristic is proposed, called Enhanced Bat Algorithm (EBA), which is developed to improve the search space exploration through some modifications in the original $\mathrm{BA}$, aiming to increase the efficiency of the search for good solutions to the large-scale ED problem.

In [14] the problem of economic dispatch is approached considering valve point effects, transmission losses and POZs, and to solve the problem a full mixed-integer linear programming implementation was proposed.

In [15] a methodology based on Ant Colony Optimization is used to minimize the daily programming cost of TPPs, which uses a Sensitivity Matrix (SM) based on information provided by the Lagrange multipliers to improve the biologically inspired search process and a certain number of individuals (ants) in the colony use this information in the evolutionary process of the colony.

Under this background, the present work proposes the use of the Nomadic People Optimizer (NPO) to solve the ED problem with prohibitive zones. The NPO is a new swarmbased metaheuristic, still little studied for application in real problems, since it was introduced in the literature recently.

The NPO simulates the nomads' behavior, who are constantly moving from place to place in search of vital resources such as water and food [16]. The nomadic people can be classified into many types, but the NPO algorithm was developed based on the Bedouins' classification and lifestyle [16]. The Sheikh family, which is the leader of the clan, and the normal families are the two types of families that composes a Bedouin clan [16]. The Sheikh leadership condition is usually hereditary, but a normal family can contest the Sheikh position in a conflict and may become the new Sheikh. The Sheikh is responsible to determine the locations that are essentials for survival and also determines the pattern of distribution for the families of the rest of the tribe, which occurs in a semicircular shape, with the Sheikh's tent at the center [16]. Also, it is function of the Sheik decide when the families will move in search of a new suitable location to ensure the survival and these families will move in different distances and directions in a randomly manner [16]. When a better location is found by a family, the clan is re-established.

In original article [16], the NPO algorithm is validated based on 36 unconstrained benchmark functions. For the comparison purpose, six well-established nature-inspired algorithms are performed for evaluating the robustness of the NPO algorithm, being particle swarm optimization (PSO)[17], Artificial Bee Colony (ABC) [18], Gray Wolf Optimizer (GWO) [19], Flower Pollination Algorithm (FFA) [20], Covariance Matrix Adaptation Evolution Strategy (CMAES) [21], and FPA), and Firefly Algorithm (FFA) [22]. The results showed that the NPO exerted superior performance.

In [23], the NPO is used for the multi-objective design of a large independent hybrid energy system composed of a photovoltaic matrix, wind turbines and energy storage in batteries. The hybrid system aims to find the best sizing of renewable energy sources with battery storage to minimize the Total Life Cycle Cost and Total Dump Energy, over the project life cycle for 25 years for a complex with 30 in Thi-Qar, located in southern Iraq. These are the only two publications present in the specialized literature.

The main contributions of this paper can be summarized as follows:

- Investigation of the NPO performance for the solution of a real power system problem, in this case the ED problem with POZs, since the NPO algorithm is new and still underexplored for real-problem solutions;

- Comparison with other metaheuristics present in the literature;

- Sensitivity analysis that evaluates the impact of the number of clans and families in the solution quality;

- Adaptation to provide NPO to work with integer variable.

The rest of this paper is organized as follows: In section II it is presented the modeling of ED problem with the inclusion of prohibitive operating zones, in section III it is introduced the NPO algorithm modeling, section IV show the study cases, the simulations, results and discussions and, finally, section $\mathrm{V}$ presents the conclusions of this work.

\section{MATHEMATICAL MODELING OF ED PROBLEM WITH PROHIBITIVE ZONES}

The economic dispatch problem consists of minimizing the system's generation cost through the optimal dispatch of thermal power plants, respecting the generation constraints of each plant that makes up the system, as well as meeting the total demand. Thus, the objective function of the problem is to minimize the sum of the generation costs of each plant that composes the system, represented by (1), where $F_{T}$ is the total generation cost of the system, $F_{i}$ is the generation cost of the i-th generator, $P_{i}$ is the power generated by the $\mathrm{i}$-th generator and $\eta_{g}$ is the number of thermal generators of the system. The constraints of the problems are presented in (2), where $P_{D}$ is the total active power demand of the system 
and $P_{i}^{\text {min }}$ and $P_{i}^{\max }$ are the minimum and maximum active power generation limits of the i-th generator.

$$
\text { Minimize } F_{T}=\sum_{i=1}^{\eta_{g}} F_{i}\left(P_{i}\right)
$$

subject to:

$$
\begin{gathered}
\sum_{i=1}^{\eta_{g}} P_{i}=P_{D} \\
P_{i}^{\text {min }} \leq P_{i} \leq P_{i}^{\text {max }}, \forall i=1, \ldots, \eta_{g}
\end{gathered}
$$

The cost function of each generator $\left(F_{i}\right)$ is related to the fuel cost of each generating unit and can be approximated by (3), as detailed in [6].

$$
F_{i}=a_{1}+b_{i} P_{i}+c_{i} P_{i}^{2}
$$

where $a_{i}, b_{i}$ e $c_{i}$ are the cost coefficients of generator $i$.

According to what has already been exposed in the present work, this ED problem modeling is not realistic. Therefore, in order to obtain a representation of the problem closer to reality, prohibitive operation zones are introduced in the model, which represent the physical limitations of the generator machines [3]. Thus, there are operating regions that are unstable and, therefore, it is necessary to avoid them [7], causing discontinuities in the plant's generation curve. With the inclusion of POZs in the ED problem, a set of constraints composed of inequalities that delimit the allowed generation regions for each generator in the system is included. These restrictions are presented in the equation 4.

$$
\begin{gathered}
P_{i}^{m i n} \leq P_{i} \leq P_{i}^{n 1} \\
P_{i}^{n 2} \leq P_{i} \leq P_{i}^{n 3} \\
P_{i}^{n 4} \leq P_{i} \leq P_{i}^{\max }
\end{gathered}
$$

where $P_{i}^{n 1}$ and $P_{i}^{n 3}$ are the upper limits and $P_{i}^{n 2}$ and $P_{i}^{n 4}$ are the lower limits of active operating power allowed by POZs.

Figure 1 illustrates a generic curve of cost versus active power generation [3]. From this figure it is possible to see the discontinuities inherent to POZs.

\section{Nomadic People Optimizer mathematiCAL MODELING}

As aforementioned, the NPO is based on the Bedouins' behavior. The NPO algorithm has five main levels, which are:

1) Initial meeting;

2) Semi-circular distribution;

3) Families searching;

4) Leadership transition;

5) Periodical meetings.

In the level one, a set of leaders is initialized by (5).

$$
\vec{\sigma}_{c}=(U B-L B) \cdot \operatorname{rand}+L B
$$

Where $\vec{\sigma}_{c}$ is the position of the leader of the clan $c, U B$ is the upper bound, $L B$ is the lower bound and rand is a random value between 0 and 1 with uniform distribution.

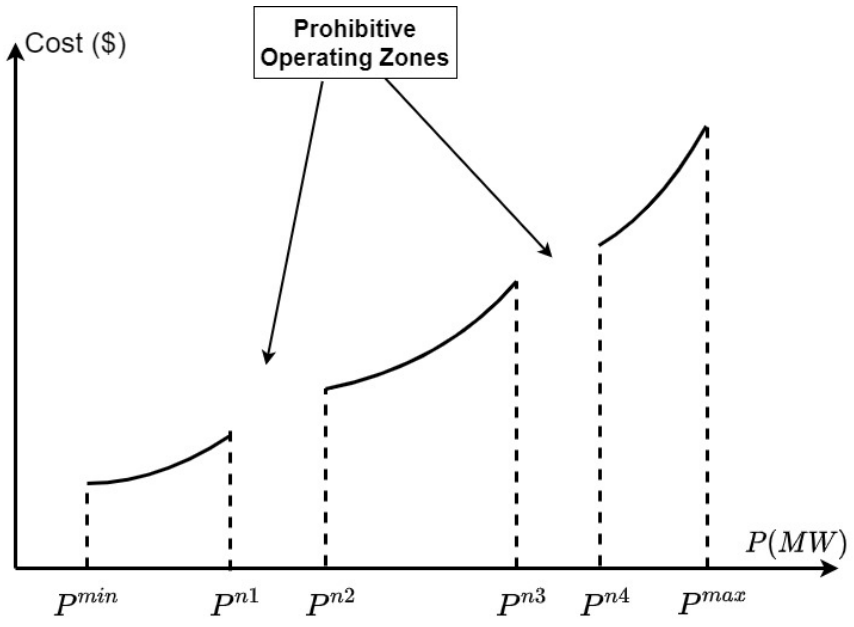

Fig. 1. Cost function of TTPS considering POZs

The semi-circular mechanism is a distribution of a set of families around the clan leader $(\sigma)$, determined by (6) [16]. Through equation 6 it can be notice that the position of the families is based entirely on the position of the leader [16].

$$
\vec{X}_{c}=\vec{\sigma}_{c} \cdot \sqrt{R} \cdot \cos (\theta)
$$

where $X_{c}$ is the family position, $R$ is a random value in the range $[0,1]$ and $\theta$ is a random angle value which respects $0 \leq \theta \leq 2 \pi$.

The families searching step is the exploration part, made up when don't have a new local best solution in the swarm, and then, the families search for better positions far away from the current local best solution. The movement of all families are determined by random directions generated by Lévy Flight formula given by (7) [16].

$$
\vec{X}_{i}^{\text {new }}=\vec{X}_{i}^{\text {old }}\left(a_{c} \overrightarrow{\left(\sigma_{c}-X_{i}^{\text {old }}\right)} \oplus \text { Levy }\right)
$$

where $\vec{X}_{i}^{\text {new }}$ represents the new family position, $\vec{X}_{i}^{\text {old }}$ represents the old family position, and $a_{c}$ is the area of the clan, obtained with (8).

$$
a_{c}=\frac{\sum_{i=1}^{\Phi} \sqrt{\left(\vec{\sigma}_{c}-\vec{X}_{i}^{\text {old }}\right)^{2}}}{\Phi}
$$

where $\Phi$ is the number of families in each clan.

The families move in different directions with random step sizes calculated by the following probability distribution [16, 24]:

$$
\text { Levy } \sim u=t^{-\lambda} \quad(1<\lambda \leq 3)
$$

The leadership transition is the stage when each family of a clan is checked and if has a family with a better fitness value than the current leader of the same clan, this family becomes a the new leader of the clan $[16,24]$.

The periodical meetings involve only the leaders and occurs for they can resolve any external problem and discuss the best 
locations for relocation, and proceeds in two stages. At the first stage occurs the determination of the overall best leader (the one with the best location). Then, this leader will provide solutions for the other Sheikhs to update their locations, which is made by adding the variance between the position of the strongest leader and the positions of the normal leader [16]. This process is described by (10).

$$
\Delta_{p o s}=\Psi\left(\frac{\sqrt{\sum_{i}^{D}\left(\sigma^{E}-\sigma_{c}^{N}\right)^{2}}}{D}\right)
$$

where $\Delta_{\text {pos }}$ denotes the normalized distance between the best and the normal leaders, $\sigma^{E}$ is the position of the best leader, $\sigma_{c}^{N}$ are the position of the normal leaders, $D$ represents the number of dimensions of the problem. $\Psi$ is the direction parameter, which guides the clan leaders to better positions, based on the fitness value of the best Sheikh, following (11) [16].

$$
\Psi=\left\{\begin{array}{cc}
1 & \text { if } f\left(\sigma^{E}\right) \geq 0 \\
-1 & \text { otherwise }
\end{array}\right.
$$

The position of the normal leaders is updated through (12), that represents a part of the NPO exploration stage [16].

$$
\vec{\sigma}_{c}^{n e w}=\vec{\sigma}_{c}^{N}+\Delta_{p o s}\left(\sigma^{E}-\sigma_{c}^{N}\right) \cdot \frac{i t e r}{T}
$$

where $\vec{\sigma}_{c}^{\text {new }}$ is the new position of the normal leader, $\vec{\sigma}_{c}^{N}$ is the old position of normal leader, iter is the current iteration and $T$ represents the total number of iterations [16].

The step-by-step of the NPO algorithm is presented hereinafter by Algorithm 1 [16].

\section{Cases, Simulations And Results}

The metaheuristics Nomadic People Optimizer (NPO), Ant Colony Optimization (ACO) [25] and Whale Optimization Algorithm (WOA) [26], are applied in two thermoelectric generation systems with prohibited zone of operation and their performances are compared. Moreover, a study is carried out on the performance of the NPO in relation to its parameters: number of clans $n C$ and number of families $n F$.

The following cases are evaluated:

- Case A: System with 10 TPPs.

- Case B: System with 40 TPPs.

- Case C: Variation of parameters inherent to the NPO.

All simulations were conducted using a Intel Core $i 7$ processor with $2.7 \mathrm{GHz}$. The codes were implemented with the software MATLAB®. 50 simulations were performed for each meta-heuristic in solving cases $\mathrm{A}$ and $\mathrm{B}$, considering 50 iterations as stopping criterion. For the ACO and WOA metaheuristics, a population of 50 individuals was adopted. The ACO pheromone evaporation coefficient $\rho$ parameter was adopted as 0.25 [25]. Data from thermoelectric systems are available in [27]. In cases $\mathrm{A}$ and $\mathrm{B}$, the number of clans $n C$ and number of families $n F$ for the NPO are used as 5 and
10 respectively, being equivalent to a total population of 50 individuals.

The applied meta-heuristics are responsible for indicating the operation zones, while the solver fmincon, present in the Optimization Toolbox of the software MATLAB®, is responsible for solving the optimal economic dispatch considering the indicated operation zones. Thus, the fitness value of individuals is provided by fmincon, where solutions that do not meet a correct convergence are penalized. The solutions provided by the meta-heuristics are integer vectors, where each component represents in which operating area the generator with operating prohibition zones will operate.

\section{A. Adaptations}

Some adaptations in the meta-heuristics were necessary to fit the problem, as the WOA and NPO meta-heuristics were initially proposed for solving continuous problems [16, 26]. As in the model used in the economic dispatch problem with prohibitive zones, the meta-heuristics are responsible for providing solutions that indicate which operating zones will be considered, representing a combinatorial problem with integer variables, the new solutions are rounded off throughout the iterative process, adapting the meta-heuristics to solve the problem. In addition, other adjustments were made to the NPO:

- In the semi-circular distribution, described in (6), the random angle $\theta$ value which respects $0 \leq \theta \leq \pi / 2$.

- In the the periodical meetings (12), the normalized distance between the best Leader and the normal leader $\Delta_{\text {pos }}$ is multiplied by 10 .

- In the Lévy Flight, the adopted step value is equal to 1.

\section{B. Case A: System with 10 TPPs}

The system with 10 thermoelectric plants supplies a load of $2700 \mathrm{MW}$, with all generators having prohibitive operating areas, where nine have three operating zones and one has two zones. Table I presents the lowest operating cost obtained by each meta-heuristic, as well as the set of operating zones referring to this cost. The standard deviation, mean, median, maximum and minimum cost are presented in the Table II.

\begin{tabular}{|c|c|c|c|c|c|}
\hline & Cost (\$) & Zone 1 & Zone 2 & Zone 3 & Zone 4 \\
\hline ACO & 652.39 & $\begin{array}{c}3,5,7 \\
10\end{array}$ & 1 & $\begin{array}{c}2,4,6 \\
8,9\end{array}$ & $\mathrm{x}$ \\
\hline WOA & 652.39 & $\begin{array}{c}3,5,7 \\
10\end{array}$ & 1 & $\begin{array}{c}2,4,6 \\
8,9\end{array}$ & $\mathrm{x}$ \\
\hline GWO & 652.39 & $\begin{array}{c}3,5,7 \\
10\end{array}$ & 1 & $\begin{array}{c}2,4,6 \\
8,9\end{array}$ & $\mathrm{x}$ \\
\hline
\end{tabular}

TABLE I

BEst SOLUTION FOR CASE A.

All metaheuristics obtained the optimal solution of 653.78 \$. The NPO obtained the optimal solution in all 50 simulations, while the WOA obtained it in 38 of the 50 simulations and the ACO in only 9 simulations. The figure 2 shows the boxplot. 


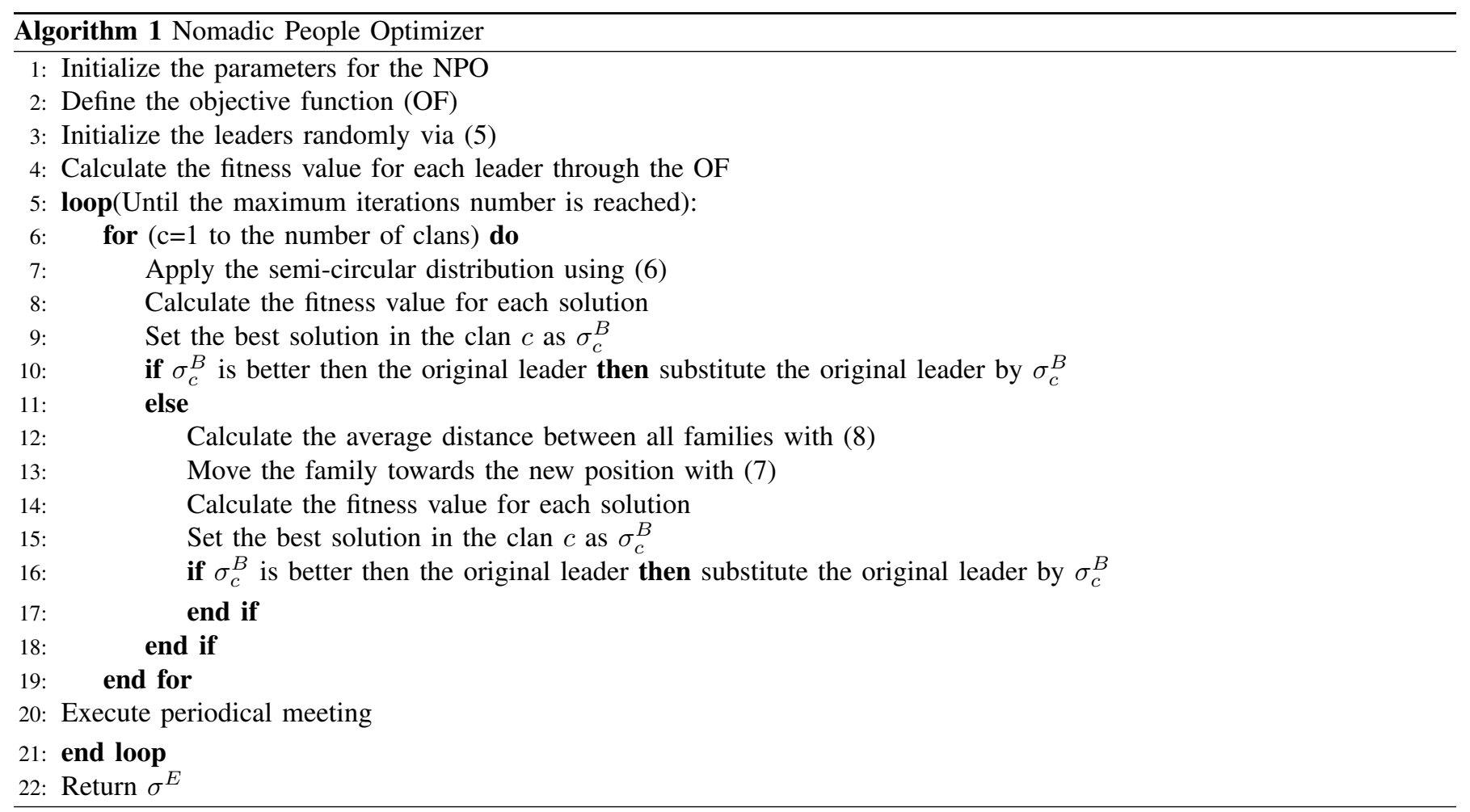

TABLE II ANALYSIS OF THE RESULTS OF CASE A.

\begin{tabular}{lccccc}
\hline & std & median & avg & max & min \\
\hline ACO & 3.00 & 655.32 & 656.12 & 662.94 & 652.25 \\
WOA & 3.96 & 652.25 & 656.12 & 671.85 & 652.25 \\
NPO & $\mathbf{0 . 0 0}$ & $\mathbf{6 5 2 . 2 5}$ & $\mathbf{6 5 2 . 2 5}$ & $\mathbf{6 5 2 . 2 5}$ & $\mathbf{6 5 2 . 2 5}$ \\
\hline
\end{tabular}

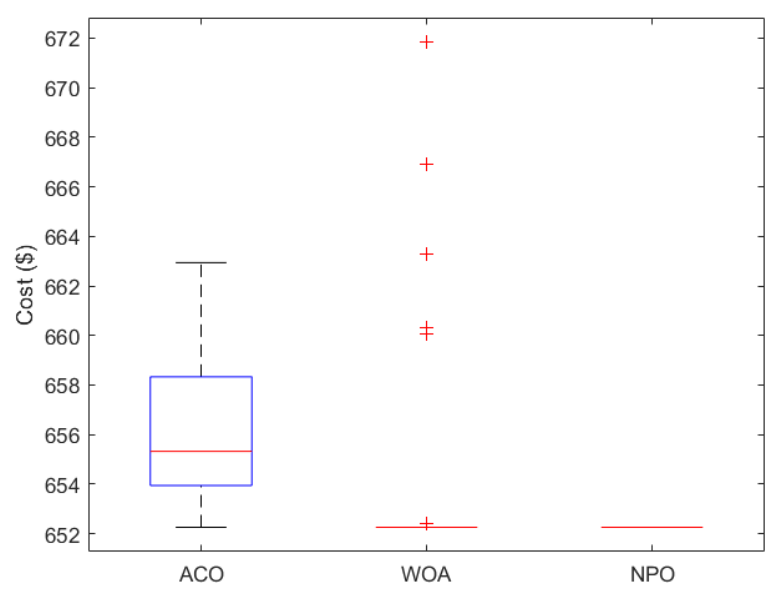

Fig. 2. Boxplot 10 TPPs

\section{Case B: System with 40 TPPs}

System with 40 TPPs, with 25 of these generators having prohibitive operation zones, with four units having four oper- ation zones, ten units having three operation zones and nine units having two. The thermal power plants with ZOPs are: 2 , $3,7,9,10,11,12,13,14,15,16,17,18,19,20,21,22,23$, $24,25,26,27,28,29$ and 30 . The demand to be met is 7000 MW. This system leads to an explosive combinatorial number of solutions, which prevents an exhaustive evaluation, so a meta-heuristic approach is justified. Table III presents the best result obtained by each meta-heuristic over the 50 simulations, and Table IV informs the standard deviation, mean, median, cost maximum and minimum obtained.

TABLE III

BEST SOLUTION FOR CASE B.

\begin{tabular}{|c|c|c|c|c|c|}
\hline & Cost $(\$)$ & Zone 1 & Zone 2 & Zone 3 & Zone 4 \\
\hline ACO & 99851.37 & $\begin{array}{c}2,3,7, \\
10,11,12, \\
13,15,16, \\
17,21,28, \\
29,30\end{array}$ & $\begin{array}{c}3,9,19, \\
20,23,25\end{array}$ & $\begin{array}{c}14,18,21, \\
22,24,26, \\
27\end{array}$ & $\mathrm{x}$ \\
\hline WOA & 99340.61 & $\begin{array}{l}2,3,7, \\
10,11,12, \\
13,14,15, \\
16,17,21, \\
28,29,30\end{array}$ & $\begin{array}{c}3,9,18 \\
20\end{array}$ & $\begin{array}{c}19,22,23, \\
24,25,26, \\
27\end{array}$ & $\mathrm{x}$ \\
\hline NPO & 99330.30 & $\begin{array}{c}2,3,7, \\
10,11,12, \\
13,14,15, \\
16,17,28, \\
29,30\end{array}$ & $\begin{array}{c}9,18,19 \\
20,21\end{array}$ & $\begin{array}{l}22,23,24 \\
25,26,27\end{array}$ & $\mathrm{x}$ \\
\hline
\end{tabular}

The NPO again achieved the best performance. Figure 3 presents the boxplot. It is important to highlight that the dispersion of the NPO results is much lower in comparison 
TABLE IV

ANALYSIS OF THE RESULTS OF CASE B.

\begin{tabular}{lccccc}
\hline & std & median & avg & max & min \\
\hline ACO & 195.64 & 10042.31 & 10036.12 & 100929.28 & 99851.37 \\
WOA & 272.35 & 99590.23 & 99673.64 & 100491.97 & 99340.61 \\
NPO & $\mathbf{2 0 . 8 6}$ & $\mathbf{9 9 3 6 4 . 4 6}$ & $\mathbf{9 9 3 5 6 . 9 0}$ & $\mathbf{9 9 3 8 1 . 2 3}$ & $\mathbf{9 9 3 3 0 . 3 0}$ \\
\hline
\end{tabular}

with the dispersions obtained by the metaheuristics WOA and ACO, which shows its superiority in solving the problem.

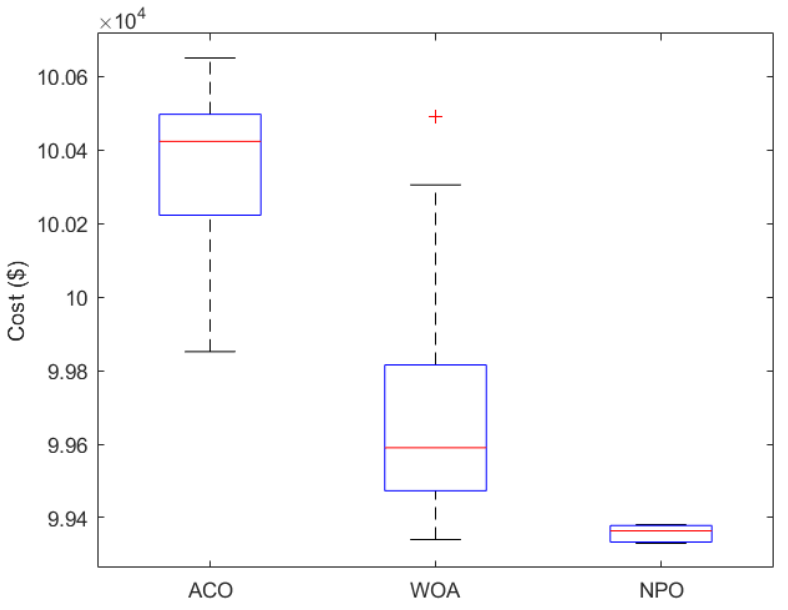

Fig. 3. Boxplot 40 TPPs

\section{Case C: Sensitivity Analysis}

Aiming to observe the influence of the number of clans, $n C$, and families, $n F$, and which of these parameters has the greatest impact on the performance in the problem solution, was performed a sensitivity analysis. For this analysis, the total number of search agents was considered as constant. In other words, the aim is verify which is more advantageous: a larger number of clans with a smaller number of families in each, or a smaller number of clans with a larger number of families in each clan. Thus, simulations are made for 7 arrays of these parameters for the system solution with 40 TPPs. The arrangements are adjusted in order to opt for a population equivalent to 100 search agents. For each parameter arrangement, 20 simulations were performed considering 25 iterations as stopping criterion. The parameter arrays, standard deviation, mean, median, highest cost and lowest cost are shown in the table $\mathrm{V}$.

Arrangement 1, arrangement with the highest number of clans $n C=50$ and $n F=2$, obtained a lower dispersion of results in the 20 simulations performed. Arrangements 3 and 7 obtained the best solution found in the literature [3]. Overall, the arrangements had good performances, which again demonstrates the success in adapting the NPO to solve the problem. The Table VI presents the best solution found in the
TABLE V

ANALYSIS OF THE RESULTS OF CASE C.

\begin{tabular}{cccccccc}
\hline$\#$ & $\mathbf{n C}$ & $\mathbf{n F}$ & std & median & avg & max & min \\
\hline $\mathbf{1}$ & 50 & 2 & $\mathbf{1 6 . 4 4}$ & 99384.69 & 99384.32 & $\mathbf{9 9 4 0 5 . 0 9}$ & 99344.90 \\
$\mathbf{2}$ & 2 & 50 & 21.19 & 99374.47 & 99362.29 & 99383.38 & 99333.64 \\
$\mathbf{3}$ & 25 & 4 & 21.17 & $\mathbf{9 9 3 6 7 . 8 5}$ & 99359.20 & 99383.38 & $\mathbf{9 9 3 2 2 . 8 5}$ \\
$\mathbf{4}$ & 4 & 25 & 18.16 & 99372.98 & 99366.03 & 99385.31 & 99333.64 \\
$\mathbf{5}$ & 20 & 5 & 20.76 & 99372.98 & 99363.85 & 99397.09 & 99330.30 \\
$\mathbf{6}$ & 5 & 20 & 22.34 & $\mathbf{9 9 3 6 7 . 8 5}$ & 99360.13 & 99393.00 & 99324.83 \\
$\mathbf{7}$ & 10 & 10 & 20.93 & 99370.50 & $\mathbf{9 9 3 5 5 . 2 8}$ & 99378.63 & $\mathbf{9 9 3 2 2 . 8 5}$ \\
\hline
\end{tabular}

sensitivity analysis, and Figure 4 shows the boxplot of the results.

TABLE VI

BEST SOLUTION FOUND IN THE SENSITIVITY ANALYSIS.

\begin{tabular}{ccccc}
\hline Cost $\mathbf{( \$ )}$ & Zone 1 & Zone 2 & Zone 3 & Zone 4 \\
\hline & $2,3,7$, & & & \\
& $10,11,12$, & $3,9,18$, & $22,23,24$, & \\
99322.85 & $13,14,15$, & $19,20,21$, & $25,26,27$ & \\
& $16,17,21$, & 25 & & \\
& $28,29,30 \backslash$ & & & \\
\hline
\end{tabular}

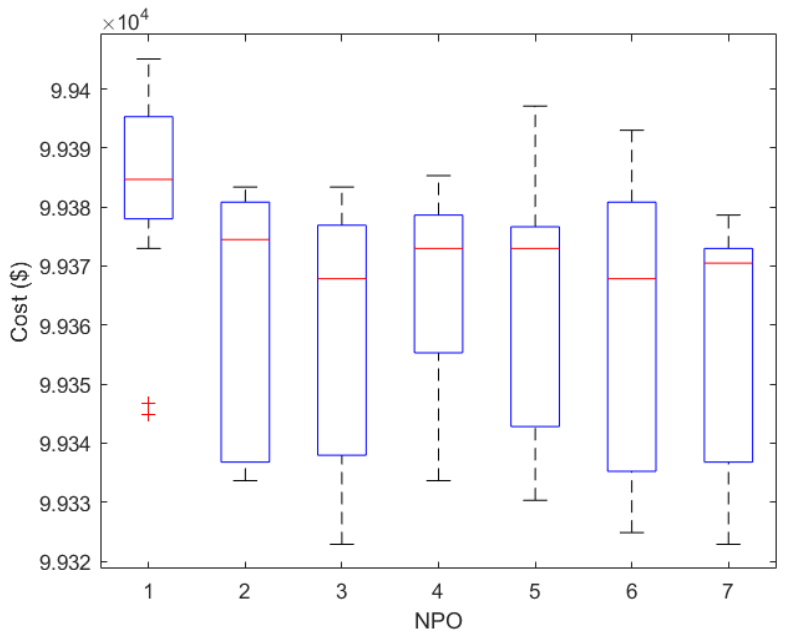

Fig. 4. Boxplots sensitivity analysis.

\section{CONCLUSION}

This work adapted the Nomadic People Optimizer (NPO), to solve the problem of economic dispatch of thermoelectric generators with prohibitive operating zones. Two thermoelectric generation systems were used in order to validate the NPO usage. From the results, some points can be emphasized:

- The performance of NPO was compared with Ant Colony Optimization (ACO) and Whale Optimization Algorithm (WOA) meta-heuristics, where NPO proved to be superior to solve the problem;

- A sensitivity analysis of the number of clans and number of families were performed showing that there was no 
significant variation in the quality of the solution due to the high efficiency of the proposed method, regardless of the number of families and clans, solving the problem of ED with POZs in a very satisfactory way;

- It can be stated that the proposed adaptation was successful in solving the ED with POZs, proving to be a competitive tool for solving the problem.

Finally, the NPO showed be suitable to be applied in a real power system. As future work, the authors aiming to evaluate the NPO method for a higher complexity level problem related to power systems and perform a sensitivity analysis to verify if in more complex problems the number of families and clans, considering the number of search agents constant, has a significant impact in the solution quality.

\section{ACKNOWLEDGMENT}

The authors acknowledge UFJF, FAPEMIG, CNPq, INERGE, GOPT-UFJF and GOHB-UFJF for their support.

\section{REFERENCES}

[1] Operador Nacional do Sistema Elétrico-ONS. Boletim diário da operação. http://sdro.ons.org.br/SDRO/ DIARIO/index.htm, 2021. [Online; accessed 29-Jun2021].

[2] Erasmo Antonio Dal'orto, Edmarcio Belati, Leonardo Willer de Oliveira, and Ivo Chaves Silva Junior. Despacho de termoelétricas via otimização clássica considerando zonas proibitivas de operação. Simpósio Brasileiro de Sistemas Elétricos-SBSE, 1(1), 2020.

[3] Ezequiel S Oliveira, Ivo C Silva Junior, Leonardo W Oliveira, Bruno H Dias, and Manuela Filgueiras. Despacho econômico com zonas de operação proibidas através de otimização bioinspirada.

[4] Flávia R Nascimento, Ivo C Silva, Edimar J Oliveira, Bruno H Dias, and André LM Marcato. Thermal unit commitment using improved ant colony optimization algorithm via lagrange multipliers. In 2011 IEEE Trondheim PowerTech, pages 1-5. IEEE, 2011.

[5] Allen J Wood, Bruce F Wollenberg, and Gerald B Sheblé. Power generation, operation, and control. John Wiley \& Sons, 2013.

[6] Jizhong Zhu. Optimization of power system operation. John Wiley \& Sons, 2015.

[7] SO Orero and Malcolm R Irving. Economic dispatch of generators with prohibited operating zones: a genetic algorithm approach. IEE Proceedings-Generation, Transmission and Distribution, 143(6):529-534, 1996.

[8] Layon M Oliveira, Ezequiel S Oliveira, and Ivo C Silva Junior. Optimal economic dispatch of thermoelectric power units with practical constraints through an enhanced bat algorithm. Anais da Sociedade Brasileira de Automática, 2(1), 2020.

[9] JY Fan and JD McDonald. A practical approach to real time economic dispatch considering unit's prohibited operating zones. IEEE transactions on power systems, 9(4):1737-1743, 1994.

[10] Fred Glover. Future paths for integer programming and links to artificial intelligence. Computers \& Operations Research, 13(5):533 - 549, 1986. Applications of Integer Programming.

[11] Gianfranco Chicco and A. Mazza. Heuristic optimization of electrical energy systems: Refined metrics to compare the solutions. Sustainable Energy, Grids and Networks, 17:100197, 022019.

[12] Mohamed Abdel-Basset, Laila Abdel-Fatah, and Arun Kumar Sangaiah. Chapter 10 - metaheuristic algorithms: A comprehensive review. In Arun Kumar Sangaiah, Michael Sheng, and Zhiyong Zhang, editors, Computational Intelligence for Multimedia Big Data on the Cloud with Engineering Applications, Intelligent Data-Centric Systems, pages 185 - 231. Academic Press, 2018.

[13] Hamdi Abdi, Hamid Fattahi, and Sara Lumbreras. What metaheuristic solves the economic dispatch faster? a comparative case study. Electrical Engineering, 100, 12 2018.

[14] Shanshan Pan, Jinbao Jian, Huangyue Chen, and Linfeng Yang. A full mixed-integer linear programming formulation for economic dispatch with valve-point effects, transmission loss and prohibited operating zones. Electric Power Systems Research, 180:106061, 2020.

[15] Ivo C Silva Jr, Flavia $\mathrm{R}$ do Nascimento, Edimar $\mathrm{J}$ de Oliveira, Andre LM Marcato, Leonardo W de Oliveira, and Joao A Passos Filho. Programming of thermoelectric generation systems based on a heuristic composition of ant colonies. International Journal of Electrical Power \& Energy Systems, 44(1):134-145, 2013.

[16] Sinan Q Salih and AbdulRahman A Alsewari. A new algorithm for normal and large-scale optimization problems: Nomadic people optimizer. Neural Computing and Applications, 32(14):10359-10386, 2020.

[17] J. Kennedy and R. Eberhart. Particle swarm optimization. In Proceedings of ICNN'95 - International Conference on Neural Networks, volume 4, pages 1942-1948 vol.4, 1995.

[18] Dervis Karaboga and Bahriye Akay. A comparative study of artificial bee colony algorithm. Applied Mathematics and Computation, 214:108-132, 082009.

[19] Seyedali Mirjalili, Seyed Mohammad Mirjalili, and Andrew Lewis. Grey wolf optimizer. Advances in Engineering Software, 69:46 - 61, 2014.

[20] Yang XS. Unconventional Computation and Natural Computation, 7445, 2012.

[21] Nikolaus Hansen, Sibylle D. Müller, and Petros Koumoutsakos. Reducing the time complexity of the derandomized evolution strategy with covariance matrix adaptation (cma-es). Evolutionary Computation, 11(1):118, 2003.

[22] Nur Johari, Azlan Zain, Noorfa Mustaffa, and Amir- 
mudin Udin. Firefly algorithm for optimization problem. Applied Mechanics and Materials, 421, 042013.

[23] Abbas Mohammed, Kassim al anbarri, and Rafid Hannun. Introducing newly developed nomadic people optimizer (npo) algorithm to find optimal sizing of a hybrid renewable energy. IOP Conference Series: Materials Science and Engineering, 928:022052, 112020.

[24] Abbas Q Mohammed, Kassim A Al-Anbarri, and Rafid M Hannun. Optimal combination and sizing of a stand-alone hybrid energy system using a nomadic people optimizer. IEEE Access, 8:200518-200540, 2020.

[25] Marco Dorigo. Optimization, learning and natural algorithms. Ph. D. Thesis, Politecnico di Milano, 1992.

[26] Seyedali Mirjalili and Andrew Lewis. The whale optimization algorithm. Advances in Engineering Software, 95:51 - 67, 2016.

[27] Ram Naresh, Jayant Dubey, and Jagannath Sharma. Twophase neural network based modelling framework of constrained economic load dispatch. Generation, Transmission and Distribution, IEE Proceedings-, 151:373 378, 062004. 